\title{
Multiple choice questions in evidence based medicine
}

\author{
Wai-Ching Leung
}

\section{Questions 1-3}

A psychiatrist devised a short screening test for depression. An independent blind comparison was made with a gold standard for diagnosis of depression among 200 psychiatric outpatients. Among the 50 outpatients found to be depressed according to the gold standard, 35 patients were positive for the test. Among 150 patients found not to be depressed according to the gold standard, 30 patients were found to be positive for the test.

1. Corrected to the nearest decimal place:

(A) The sensitivity was $80 \%$

(B) The specificity was $80 \%$

(C) The positive predictive value was $70 \%$

(D) The negative predictive value was $88.9 \%$

(E) The prevalence of depression was $25 \%$

A general practitioner decides to use the screening test for his patients. The prevalence of depression among his patients is estimated to be $5 \%$.

2. The following measures are identical whether the test is applied to general practice patients or to the psychiatric outpatients:

(A) Prevalence of depression

(B) Sensitivity

(C) Specificity

(D) Positive predictive value

(E) Negative predictive value

Northern Region

Public Health

Medicine Training

Scheme

Correspondence to: Dr Wai-Ching Leung, Epidemiology and Public Health, 1st floor, Milvain Building, Newcastle General Hospital, Westgate Road, Newcastle-upon-Tyne

NE4 6BE, UK (email: Wai_chingleung@hotmail.com)

Submitted 1 February 2000 Accepted 19 April 2000
3. Applying to a patient seen in general practice with a positive test:

(A) The pre-test odds for depression is 1 in 20

(B) The pre-test probability for depression is 1 in 19

(C) The likelihood ratio for a positive test is 3.5

(D) The post-test odds for depression is 7 in 38

(E) The post-test probability for depression is 7 in 45

\section{Questions 4-7}

A total of 360 patients participated in a randomised controlled trial designed to compare the effectiveness of drug $\mathrm{X}$ in reducing deaths with a placebo. Out of 120 patients in the treatment group, 12 patients died within three years. Out of 240 patients in the control group, 48 patients died within three years.

4. The following measures for the risk of death within three years (correct to the nearest 1 decimal place) are true:

(A) The event rate in the control group is 0.3

(B) The event rate in the treatment group is 0.1

(C) The relative risk (of treatment compared with control group) is 0.25

(D) The relative risk reduction is $67 \%$ (to the nearest \%)

(E) The number needed to treat to prevent 1 death is 10

5. The following measures used for evaluating the level of clinical significance are independent of the patient's expected event rate:

(A) Odd ratios

(B) Relative risks

(C) Relative risk reduction

(D) Absolute risk reduction

(E) Numbers needed to treat

6. In a randomised controlled trial, the following types of biases are reduced by randomisation:

(A) Ascertainment bias

(B) Selection bias

(C) Recall bias

(D) Publication bias

(E) Bias in handling dropouts

7. The conclusions of a meta-analysis may be rendered invalid by:

(A) Clinical heterogeneity

(B) Statistical heterogeneity

(C) Database bias

(D) Symmetrical funnel plot

(E) English language bias 
Table 1 A $2 \times 2$ table

\begin{tabular}{clrr}
\hline \multicolumn{4}{c}{ Depression } \\
\cline { 2 - 3 } & Present & Absent & \\
\hline Test & & & \\
+ & 35 & 30 & 65 \\
- & 15 & 120 & 135 \\
& 50 & 150 & 200 \\
\hline
\end{tabular}

Answers

1. (A) false, (B) true, (C) false, (D) true, (E) true

A $2 \times 2$ table can be constructed as shown in table 1 .

Sensitivity $=$ the proportion of subjects with the disease correctly diagnosed by the test = $35 / 50=70 \%$. Specificity $=$ the proportion of subjects without the disease correctly excluded by the test $=120 / 150=80 \%$. Positive predictive value $=$ the proportion of subjects with a positive test who have the disease = $35 / 65=53.8 \%$. Negative predictive value $=$ the proportion of subjects with a negative test who do not have the disease $=120 / 135=88.9 \%$. Prevalence of depression $=50 / 200=25 \%$

2. (A) false, (B) true, (C) true, (D) false, (E) false

The prevalence of depression in general practice $(5 \%)$ is lower than among the psychiatric outpatients (25\%). Sensitivity and specificity are characteristics of a diagnostic test and do not change with prevalence of the population. Positive predictive value increases with the prevalence, while negative predictive value decreases with the test.

3. (A) false, (B) false, (C) true, (D) true, (E) true

Odds is the ratio of events to non-events. A prevalence of $5 \%$ means that for every patient who is depressed, there are 19 non-depressed patients. Hence, the pre-test odds is 1 in 19 . The pre-test probability is 1 in 20 . The likelihood ratio for a positive test is a characteristic of the test and does not change with prevalence. Likelihood ratio $=$ sensitivity $/$ $(1-$ specificity $)=70 \% /(1-80 \%)=3.5$.

Post-test odds $=$ pre-test odds $\times$ likelihood ratio for positive test $=1 / 19 \times 3.5=7$ in 38 . Hence, post-test probability $=7$ in $(7+38)=7$ in 45 .

4. (A) false, (B) true, (C) false, (D) false, (E) true

Event rate in the control group $(\mathrm{CER})=$ $48 / 240=0.2$.

Event rate in the experimental group $(\mathrm{EER})=$ $12 / 120=0.1$.

Absolute risk reduction $(\mathrm{ARR})=\mathrm{CER}-\mathrm{EER}$ $=0.2-0.1=0.1$.

Relative risk reduction $(\mathrm{RRR})=\mathrm{ARR} / \mathrm{CER}$ $=0.1 / 0.2=50 \%$.

Numbers needed to treat $(\mathrm{NNT})=1 / \mathrm{ARR}$ $=10$.
5. (A) true, (B) true, (C) true, (D) false, (E)

false

Odds ratio, relative risk, and relative risk reduction are independent of the patient's expected event rate. These measures quoted in studies are directly applicable to any group of patients. However, the absolute risk reduction increases, while the numbers needed to treat decreases, with patient's expected event rate.

6. (A) false, (B) true, (C) false, (D) false, (E)

false

Subjects are randomised in a randomised controlled trial to ensure that the groups are identical apart from the intervention. Randomisation means that each subject has a predetermined chance of being allocated to each group, but the group to be allocated cannot be predicted. This will minimise any selection bias. Ascertainment bias occurs when the results of a trial are systematically distorted by knowledge of which intervention each participant is receiving. This type of bias is minimised by blinding of researchers, clinicians, and patients. Bias caused by dropouts occur because characteristics of subjects who left the trial before completion may be different from those who do not. This type of bias is minimised by appropriate analysis (for example, intention to treat analysis, worst case scenario analysis). Recall bias occurs when the subjects' recall of past events is distorted by subsequent events. This type of bias occurs mainly in retrospective studies (for example, case control studies) rather than randomised controlled trials. Publication bias occurs during the dissemination of the results.

\section{7. (A) true, (B) true, (C) true, (D) false, (E)} true

The conclusions of a meta-analysis may be rendered invalid if the results in the primary trials are statistically incompatible with one another (statistical heterogeneity) or if the research participants differ significantly from one another (clinical heterogeneity). If statistical or clinical heterogeneity occur, the results in the primary trials cannot be validly combined together mathematically. Bias may also occur if the selection of primary studies is incomplete and biased. This occurs mainly because compared with studies with negative results, studies with positive results are more likely to be published, published in English, and appear in databases. The funnel plot may be used to assess the presence of publication bias. ${ }^{1}$ The sample size of each primary study is plotted against its effect size. An asymmetrical funnel plot suggests that publication bias is present.

1 Egger $M$, Smith GD, Schneider $M$, et al. Bias in meta-analysis detected by a simple, graphical test. $B M \mathcal{F}$ 1997;315:629-34. 